\title{
OBSERVATIONS IN CENTRAL AND SOUTHERN EUROPE
}

\author{
V. PORUBC̆AN \\ Astronomical Institute, Slovak Academy of Sciences \\ Dúbravská 9, 84228 Bratislava, Slovakia
}

Since September 1996 there has been in operation a new forward-scatter system for meteor observation transmitting signal simultaneously along two mutually almost rectangular baselines with the transmitter at Budrio $\left(\phi_{B}=44.6^{\circ} \mathrm{N} ; \lambda_{B}=11.5^{\circ} \mathrm{E}\right)$ near Bologna, Italy and the receivers at Modra $\left(\phi_{L}=48.3^{\circ} \mathrm{N} ; \lambda_{L}=17.3^{\circ} \mathrm{E}\right)$, Slovakia and Lecce $\left(\phi_{L}=40.3^{\circ} \mathrm{N} ; \lambda_{L}=18.2^{\circ} \mathrm{E}\right)$ in Southern Italy. The equipment utilizes a continuous wave transmitting frequency at $42.7 \mathrm{MHz}$, with a mean power of $0.25 \mathrm{~kW}$.

Meteor activity in November 1996 was monitored at both the receiving stations in the period Nov. 12-26. The Leonid activity is clearly discernible only for long duration echoes and is apparent already from Nov. 13. The data exhibit a rather flat maximum lasting from about 01 UT till 10 UT, Nov. 17 with two peaks (obtained by compounding Modra and Lecce data) at approx. $235.07^{\circ}$ and $235.27^{\circ}(2000.0)$. The peaks are consistent with the compound visual data proposing also two Leonid peaks at $235.15^{\circ}$ and $235.37^{\circ}$. The 1995 Leonid observations were carried out only on the baseline Bologna - Lecce, with the transmitter working at a higher power of $1 \mathrm{~kW}$ and exhibit a multiple shower maximum too, with three peaks at $235.35^{\circ}, 235.49^{\circ}$ and $235.57^{\circ}$. The second peak is identical with the maximum obtained from the 1995 visual observations.

The mass distribution exponent found for the 1996 shower maximum (Modra and Lecce together) and echoes of duration $\geq 0.3 \mathrm{~s}$ is 1.51 . The value is confirming a high proportion of long duration echoes in the 1996 Leonids, while the sporadic background reached its standard value of $s$ $=2.28$. The mass exponent found for the shower echoes of $\geq 1 \mathrm{~s}$ is 1.56 . The mass exponent derived for the Leonids 1995 (Lecce data) was found equal to $1.67,1.69$ and 2.24 for the shower echoes of duration $\geq 0.3 \mathrm{~s}, \geq 1 \mathrm{~s}$ and the sporadic background, respectively.

A comparison of both Leonid returns shows a higher proportion of larger particles in the 1996 return with respect to the 1995 return. 